\title{
Effects of intrahippocampal injections of somatostatin and cysteamine on spatial discrimination learning in mice
}

\author{
JEAN-LOUIS GUILLOU, JACQUES MICHEAU, and ROBERT JAFFARD \\ Laboratoire de Neurosciences Comportementales et Cognitives, Université de Bordeaux I, France
}

\begin{abstract}
The involvement of hippocampal somatostatin (SS-14) in spatial discrimination learning in mice was evaluated by bilaterally administering either SS-14 or its depletor, cysteamine, into the hippocampus through chronically implanted guide cannulae. Two separate experiments were performed using an eight-arm radial maze. In Experiment 1, mice were trained for 4 days on a discrimination task in which only three arms (A, B, and C) were used. In each daily session, subjects were each presented in 10 trials with the pair $\mathrm{AB}$ and the pair $\mathrm{BC}$, with arm $\mathrm{B}$ consistently baited over trials (e.g., A-B + and B $+\mathrm{C}-$ ). Immediately after completion of the fourth daily session with these regular trials, they were given 10 additional probe trials with free access to all three arms (e.g., $\mathrm{A}-\mathrm{B}+\mathrm{C}-$ ). In Experiment 2, animals had free access to all eight arms. They were trained to discriminate the three arms that were consistently baited over trials ( 8 trials per day for 7 days). Intrahippocampal injections were given each day, $30 \mathrm{~min}$ before testing. The results of both experiments showed that, compared with saline, SS-14 administration $(0.2 \mu \mathrm{g} / 0.2 \mu \mathrm{l}$ per hippocampus) resulted in faster acquisition rates. In contrast, animals treated with cysteamine $(20 \mu \mathrm{g})$ were dramatically impaired. In Experiment 1, however, the SS-14 treatment group was significantly impaired relative to both the saline and the intact control groups when testing was shifted from regular to probe trials. The impaired ability of somatostatin-treated mice to adapt to probe trials, together with their improved performance on both types of spatial discrimination task, suggests that this peptide produced a change in the strategy the animals used to solve these tasks. Notwithstanding, the present findings seem difficult to reconcile with current theories about hippocampal function.
\end{abstract}

The tetradecapeptide somatostatin SS-14 was first isolated from ovine hypothalamus and was characterized as inhibiting the pituitary secretion of growth hormone (Brazeau et al., 1973). However, the widespread but regionally selective distribution of SS-14 neurons suggests that SS-14 is not only a hypothalamic regulator of neuroendocrine function but that it may also function as a major neuromodulator mediating a variety of functions throughout the central nervous system (Epelbaum, Brazeau, Tsang, Browxer, \& Martin, 1977; Finley, Madedrut, Roger, \& Petrusz, 1981). Many SS-immunoreactive cell bodies and processes have been observed in all regions of the cerebral cortex (Iritani \& Satoh, 1991)-namely, the frontal, parietal, temporal, insular, and occipital cingulate cortices, as well as the enthorhinal cortex, which displayed the highest SS-receptor density (Katayama, Kito, Miyoshi, \& Yamamura, 1990). Also rich in SS-14 and SS-receptors are large areas of the limbic system, notably the hippocampus (McCarty \& Plunkett, 1987; Reubi \& Maurer, 1985), where SS-14 positive cells show a preferential distribu-

This study was supported by the Centre National de la Recherche Scientifique and by the Conseil Régional d'Aquitaine. J.L.G. holds a fellowship from the Ministère de la Recherche. Correspondence and reprint requests should be sent to Jean-Louis Guillou, Laboratoire de Neurosciences Comportementales et Cognitives, URA 339, Avenue des Facultés, 33405 Talence Cedex, France. tion in the stratum oriens and stratum pyramidalae of the Ammon's horn, and match the description of the polymorphic cells of the area dentata (Köhler \& Chan-Palay, 1982). About $90 \%$ of SS-neurons are also GAD-like immunoreactive, and about $30 \%$ of the GABA-ergic neurons are also somatostatinergic in the hilus of the dentate gyrus and the stratum oriens of the CA1 and CA3 regions (Kosaka, Wu, \& Benoit, 1988). However, the physiological functions of SS-14 in the hippocampus are still unclear. Both SS-induced depolarizations and hyperpolarizations of CA1 pyramidal neurons have been described, and the involvement of SS-14 in disinhibitory mechanisms has been suggested (Pittmann \& Siggins, 1981; Scharfman \& Schwartzkroin, 1988).

Since neuropathological studies disclosed a reduced content of SS-14 and a decrease in the number of SSreceptors in the cerebral cortex and the hippocampal regions of Alzheimer's patients (Beal, Mazurek, Chattha, Bird, \& Martin, 1985; Chan-Palay, 1988; Davies, Katz, \& Crystal, 1982; Rossor, Emson, Mountjoy, Roth, \& Iverson, 1980), the functional and/or pathological role of these tetradecapeptide-containing neurons has attracted considerable interest. Direct synaptic contacts between medial septal efferents and SS-neurons in the rat hippocampus have been demonstrated (Yamano \& Luiten, 1989), and this innervation of somatostatinergic hippocampal 
neurons by the septohippocampal system may be of great interest to those concerned with understanding the morphological substrates involved in the learning and memory functions of the hippocampus. GABA-A-receptor-mediated fast IPSP and GABA-B-receptor-mediated slow IPSP are both suppressed by SS-14, and thus a modulatory role of SS-14 in the induction of long-term potentiation (LTP) has been recently suggested by Xie and Sastry (1992). In the mossy fiber-CA3 pyramidal cell system, SS-14 was shown to enhance production of LTP through the intervention of cholinergic and noradrenergic neurons, but failed to affect LTP in the Schaffer collateral-CA1 pyramidal cell system (Matsuoka, Kaneko, \& Satoh, 1991b). Moreover, cysteamine-induced depletion of SS-14 (Sagar et al., 1982) has been shown to reduce the magnitude of LTP and to enhance the SS-14-induced facilitation of LTP in the CA3 area (Matsuoka, Kaneko, \& Satoh, 1991a). The latter results also suggest that SS-14 could play an important role in the memory function of the hippocampus.

Learning and memory deficits in passive and active avoidance tasks in rats have been reported following both systemic and intracerebroventricular (i.c.v.) injections of the SS-14 depletor cysteamine (Bakhit \& Swerdlow, 1986; Denoble, Hepler, \& Barto, 1989; Haroutunian, Mantin, Campbell, Tsuboyama, \& Davis, 1987). Conversely, i.c.v. injections of SS-14 have been found to facilitate the acquisition and to delay the extinction of active avoidance learning (Vécsei et al., 1984; Vécsei \& Widerlow, 1988) and to attenuate electroshock-induced retrograde amnesia of passive avoidance learning (Vécsei, Bollók, \& Telegdy, 1983). In 1976, one study reported that direct cerebral injections of SS-14 into the hippocampal formation induced several stereotyped behaviors, alteration of the sleepwaking cycle, and a frequent dissociation of the EEG from behavior, thereby disclosing a central site of action of SS-14 (Rezek, Havlicek, Hughes, \& Friesen, 1976). Since that time, no further data on the behavioral effects of intrahippocampal injections of SS-14 have been reported, to our knowledge. In the present study, therefore, we investigated the role of hippocampal somatostatin on spatial discrimination by injecting SS-14 and cysteamine directly into the hippocampus. This behavioral protocol was chosen since one main theory regarding the nature of the involvement of the hippocampus in learning and memory emphasizes its specific role in the processing of spatial information (i.e., Nadel, 1991; O'Keefe \& Nadel, 1978). More recent theories, however, propose that the structure is involved in a more general range of specific processes, of which spatial mapping is only one example (i.e., Eichenbaum, Otto, \& Cohen, 1992; Sutherland \& Rudy, 1989). Accordingly, the test procedure was also designed to take into account these latter theories involving the hippocampus in "relational" or "configural" learning processes.

\section{METHOD}

\section{Animals}

Subjects $(n=54)$ were male mice of the BALB/c strain obtained from IFFA-CREDO (Lyon, France). They were housed in a clima- tized animal room on a 12:12-h light:dark schedule with ad-lib access to food and water. At the age of 8-12 weeks, they were housed individually. In Experiment 1, naive subjects were divided randomly into four groups: an intact control group $(n=9)$, and three different treatment groups injected with saline $(n=9)$, cysteamine $(n=9)$, or somatostatin $(n=9)$. In Experiment 2, 18 different naive subjects were divided into two groups: those injected with saline $(n=9)$ and those injected with somatostatin $(n=9)$.

\section{Surgery}

The mice were implanted under general anesthesia (sodium thiopental, $70 \mathrm{mg} / \mathrm{kg}$ i.p.) with two guide cannulae $(0.4 \mathrm{~mm}$ in diameter, $8 \mathrm{~mm}$ long) aimed vertically toward the dorsal hippocampus. Guide cannulae were fixed to the skull bone with dental cement and fine bone screws. Stereotaxic coordinates were $1.7 \mathrm{~mm}$ posterior to the bregma, $\pm 1.3 \mathrm{~mm}$ to each side of the sagittal suture, and $1.1 \mathrm{~mm}$ ventral from the skull surface. Following the operation, the mice were replaced in the animal room for a recovery period of 10 days before the experimental phase began. Following sacrifice, all the guide cannulae placements were verified histologically as being correctly located above the dorsal hippocampus. The range of the injection locations is schematically represented in Figure 1 .

\section{Drug Injections}

Bilateral intrahippocampal injections of somatostatin $(0.2 \mu \mathrm{g} /$ $0.2 \mu \mathrm{l}$; Neosystem), 2-mercaptoethylamine hydrochloride [i.e., cysteamine $(20 \mu \mathrm{g} / 0.2 \mu \mathrm{l})$; Sigma], or vehicle (pyrogen-free $0.9 \%$ physological saline) were performed in freely moving mice via injection cannulae $(0.2 \mathrm{~mm}$ in diameter, $8.7 \mathrm{~mm}$ long) attached to $1-\mu l$ Hamilton syringes via polyethylene catheter tubing. The syringes were held in a constant-rate infusion pump and injection was conducted over a 3-min period. In all cases, correct injection flow rates were visually controlled. The cannulae were left in place for a further $2 \mathrm{~min}$ before removal. Injections were given $30 \mathrm{~min}$ prior to each learning session. The $\mathrm{pH}$ of the solutions injected were controlled and adjusted to normal physiological $\mathrm{pH}(7.4)$ in the brain

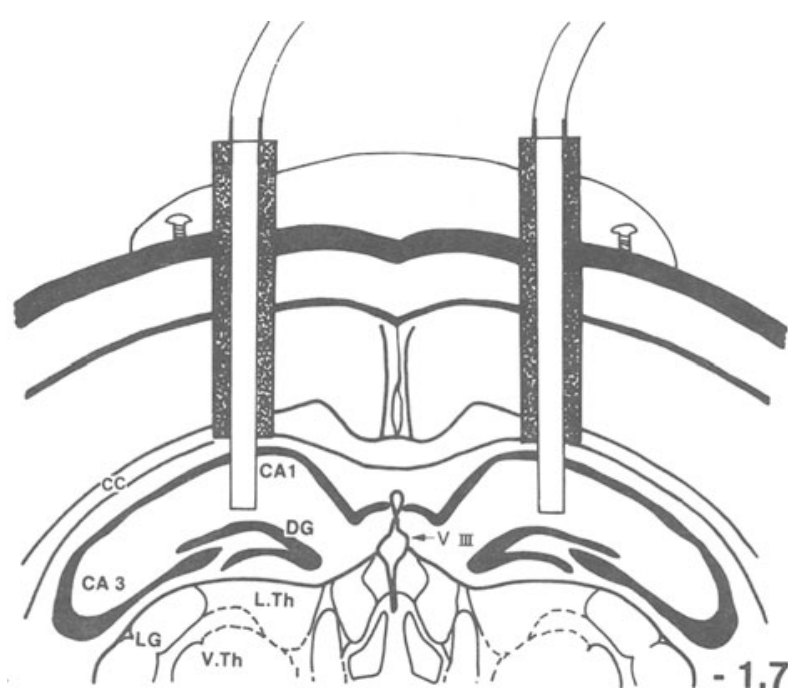

Figure 1. Diagram (frontal plane) of representative locations of both guide cannulae and injection cannulae aimed toward the dorsal hippocampus. The number in the lower right-hand corner of the section indicates the distance (in millimeters) behind the bregma. CA1 and CA3, divisions of the Ammon horn; DG, dentate gyrus; L.Th, lateral thalamic nuclei; V.Th, ventral thalamic nuclei; LG, lateral geniculate nuclei; $\mathbf{C C}$, corpus callosum; V III, 3rd ventricle. 
by addition of $\mathrm{NaOH}$, in particular because cysteamine solutions present an acidic $\mathrm{pH}$ (about 3.0).

\section{Behavioral Procedures}

Apparatus. Specific reference memory tests were conducted in an automated and elevated eight-arm radial maze based on that described by Olton, Becker, \& Handelman, (1979) and scaled down in size for use with mice. The maze was constructed of gray Plexiglas and comprised a circular central platform $(30 \mathrm{~cm}$ in diameter) from which radiated in a symmetrical fashion eight arms $(50 \mathrm{~cm}$ long $\times 11 \mathrm{~cm}$ wide). At the end of each arm was a circular food pellet tray. The maze was equipped with doors at the entrance of each arm and with photoelectric cells that detected the position of the animal. This information was constantly transmitted to a microcomputer, allowing automatic recording of arm choices, latencies, and running speeds. The microcomputer program also controlled the sequence of doors opening and closing according to the specifications of each particular test. A closed-circuit video system allowed the experimenter to visually control the test in a neighboring room.

Behavioral testing. The experimental phase began when operated mice had recovered for at least 10 days. Throughout the entire ensuing experimental period, the mice were deprived of food to maintain their body weights at $90 \%$ of their free-feeding weight.

\section{Experiment 1}

The reference memory test. The procedure used was primarily based on the ability of mice to learn more than one two-choice spatial discrimination (concurrent discrimination) by successively presenting pairs of selected adjacent arms (up to six overlapping pairs; see Jaffard et al., 1991). In the present experiment, however, only three arms (two overlapping pairs) were used in order to obtain faster acquisition rates, thereby limiting the number of daily intrahippocampal injections required. Thus, for a given subject, three adjacent arms were randomly selected: a central arm, B, always baited, and its two adjacent arms, $A$ (left-hand side) and $C$ (righthand side), that were never baited (i.e., $\mathrm{A}-, \mathrm{B}+$, and $\mathrm{C}-$ ).

Habituation and preliminary training. In order to familiarize mice with the radial maze and its environment, animals were first allowed free-exploration sessions on two successive days. During this stage, the three selected adjacent arms were baited. The three doors giving access to these arms were opened simultaneously so that the mice could freely enter each arm and find the food-pellet reward. Each daily session was terminated when all the three food pellets had been collected. The next day, animals were given 10 successive three-arm-choice trials according to the same procedure as that used at the end of the learning phase (probe trials-see below). The aim of this test was to check whether each arm was chosen at chance level $(33.3 \%$, and further, to allow a direct comparison between performances measured before and after the two-arm-choice learning phase.

Reference memory testing. During this learning phase (days $1-4)$, the animals were successively presented with each of the two pairs, $\mathbf{A}-\mathbf{B}+$ and $\mathbf{B}+\mathbf{C}-$ (regular trials). In each daily session (20 trials), each pair was presented 10 times in a pseudorandom order. The intertrial interval was $30 \mathrm{sec}$. Preliminary experiments showed that, under these conditions, normal mice displayed a significant day-to-day improvement of response accuracy, reaching scores of $75 \%-80 \%$ correct choices in the fourth session. Immediately after the completion of this fourth learning session, the animals were submitted to 10 additional three-arm-choice probe trials. On each trial, the three arms $(A-B+C-)$ were opened simultaneously.

\section{Experiment 2}

Habituation. As in Experiment 1, the mice were first allowed free exploration of the maze on 2 successive days. During this stage all doors were opened, so that the mice could freely enter each arm and find a food-pellet reward. Each daily session was terminated when all eight pellets had been collected.

Reference memory testing. Over the next 7 days, the animals learned a spatial discrimination task that is thought to specifically assess spatial mapping processes. For any 1 animal, only three out of the eight arms were constantly baited over trials, with the specification that the angles between these baited arms were $135^{\circ}-90^{\circ}$ $135^{\circ}$. The acquisition of the discrimination consisted in learning the location of the three arms. At the beginning of each trial, the eight doors were opened. After each visit to a given arm, the door giving access to that arm was automatically closed, thereby preventing the subject from making working-memory errors (i.e., reentries into an already visited arm). Each trial was terminated when all three food pellets had been collected. In each daily session, the animals were given 8 trials, each separated by a 30 -sec interval.

\section{Data analysis}

The results of Experiment 1 are expressed as the mean percentage of correct choices $( \pm S E M)$ recorded either on each 20-trial session (regular trials) or on each block of 10 trials (last 10 regular trials and probe trials). An analysis of variance (ANOVA) was used to compare the differences between groups and sessions (regular trials and probe trials), with repeated measures on sessions. Since chance performance was different for regular trials (50\%) compared with probe trials (33.3\%), a transformation of individual scores was performed to allow a direct comparison between scores obtained in each condition (see Results). Post hoc paired comparisons were made with the Scheffe $F$ test. The results of Experiment 2 are expressed as mean errors ( $\pm S E M$ ) recorded over each daily session (blocks of 8 trials). An ANOVA was used to compare the differences between groups and sessions, with repeated measures on the sessions.

\section{RESULTS}

\section{Experiment 1}

Discrimination learning (two-choice discrimination). The results are summarized in Figure 2. The ANOVA revealed a significant effect of treatments [group: $F(3,32)=20.5, p<.001]$. As a whole, there was a significant increase in choice accuracy across the four sessions [session: $F(3,96)=37.4, p<.001$ ], with a significant group $\times$ session interaction $[F(9,96)=7.6$, $p<.001]$.

Since the saline-treatment group displayed a significantly slower acquisition rate than did the intact control group [group $\times$ session interaction: $F(3,48)=5.2$, $p<.01$ ], the effects of both cysteamine and SS-14 injections on discrimination performance were evaluated against saline treatment. The results showed that, compared with saline injection, SS-14 injection speeded up acquisition $[F(3,48)=4.3, p<.01]$, leading to significantly better discrimination scores during the fourth session $(74.4 \%$ vs. $60.6 \%$ for saline; $p<.01)$. In contrast, animals injected with cysteamine were significantly impaired compared with those injected with saline (group $\times$ session interaction: $F(3,48)=3.6, p<.02$ ); in fact, the cysteamine group still exhibited chance performance $(50 \%)$ in the fourth learning session $(47.2 \% \mathrm{vs}$. $60.6 \%$ for saline; $p<.01$ ).

Probe trials (three-choice discrimination). The results are summarized in Figure 3. On the whole, discrimination scores on probe trials were significantly improved 


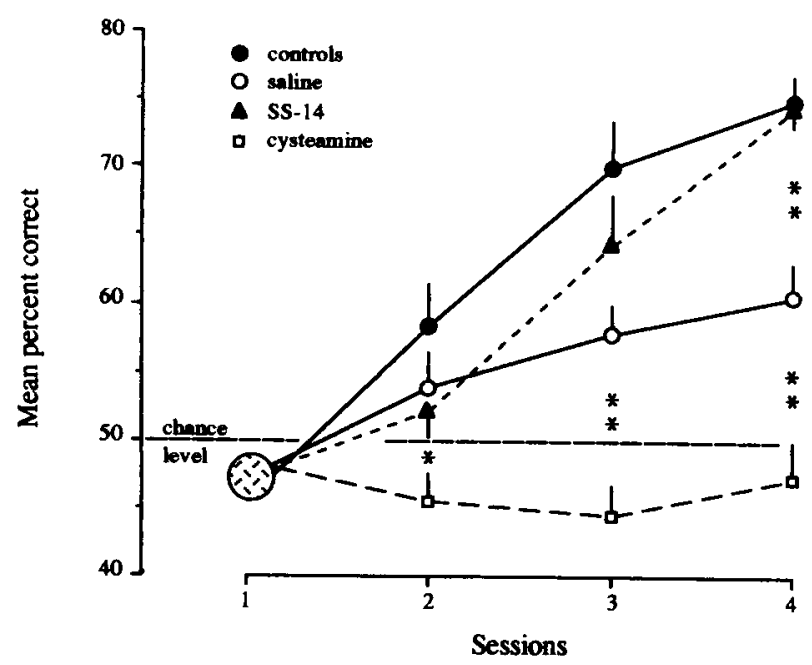

Figure 2. Performance (mean percent correct \pm SEM) following intrahippocampal injections of somatostatin (SS-14, $0.2 \mu \mathrm{g}$ ), cysteamine ( $50 \mu \mathrm{g})$, or saline, and of intact controls ( 9 animals per group), across four sessions of regular two-choice discrimination trials. * Significantly different from the saline-injected group $(p<.05$; * Significantly different from the saline-injected group $(p<.01$; post-hoc Scheffe $F$ test).

compared with those observed during the prelearning test- -that is, before the learning phase with regular twoarm-choice trials began [session: $F(1,32)=82.3, p<$ $.001]$. Subsequent analysis revealed once again that salineinjected mice displayed less improvement than did intact controls $[F(1,16)=4.1, p=.06]$ and significantly more improvement than did the cysteamine group $[F(1,16)=$ $8.4, p=.01]$. However, contrary to what could be expected on the basis of discrimination performances recorded on regular trials, the SS-14 treatment group did not exhibit better (in fact, it exhibited slightly lower) response accuracy than did the saline group when tested on the probe (three-arm-choice) trials $(55.6 \%$ vs. $66.7 \%$; $p>.05$ ). In order to allow a direct comparison between discrimination scores recorded on probe trials (chance level $=33.3 \%$ ) and regular trials (chance level $=50 \%$ ), individual data obtained on the last two-choice 10-trial block (fourth session) were transformed according to the following formula: percentage correct transformed = $100 \times n \mathrm{~B} /(n \mathrm{~B}+2 n \mathrm{~A}+2 n \mathrm{C})$, where $n \mathrm{~B}$ was the number of correct choices, and $n \mathrm{~A}$ and $n \mathrm{C}$ the number of incorrect choices, recorded on this last 10-trial block of regular trials. A two-way ANOVA showed a significant interaction between group and trial type [i.e., regular vs. probe; $F(3,32)=4,82, p<.01]$.

Thus, whereas discrimination scores (chance level $=$ $33.3 \%$ ) of both the saline and the intact control groups continued to increase between the last block of regular trials and the probe trials (from $50.5 \%$ to $66.7 \%$ and from $70.9 \%$ to $92.2 \%$, respectively), an inverse pattern of changes was actually observed in the SS-14 group [from
$69.7 \%$ to $55.6 \%$; group $\times$ block interaction: $F(1,16)>$ $6.0, p<.03$ for both comparisons].

\section{Experiment 2}

The results are summarized in Table 1. An ANOVA revealed a near-significant effect of treatments [group: $F(1,16)=3,45, p<.08]$. There was a significant decrease in errors across the seven sessions [session: $F(6,96)$ $=40.4, p<.001]$, with a significant group $\times$ session interaction $[F(6,96)=2.5, p<.02]$. Between-groups comparisons showed that the SS-14 treatment group displayed significantly fewer errors on Sessions 3 and 4 (22.1 vs. $29.8, p<.001$; and 16.7 vs. $22, p<.05$, respectively). The performance of intact mice, not trained at the same time but routinely tested to monitor this testing procedure, is also shown in Table 1. Again, compared with this intact group, the saline group displayed a lower rate of acquisition.

\section{DISCUSSION}

The results of Experiment 1 can be summarized as follows:

1. Compared with saline injections, intrahippocampal injections of somatostatin (SS-14) facilitated, whereas its depletor, cysteamine, impaired, the concurrent acquisition of a spatial discrimination composed of two separate but overlapping pairs of arms (i.e., in regular trials: $\mathrm{A}-\mathrm{B}+$ and $\mathrm{B}+\mathrm{C}-)$. In addition, compared with the intact control group, the rate of acquisition in the saline-

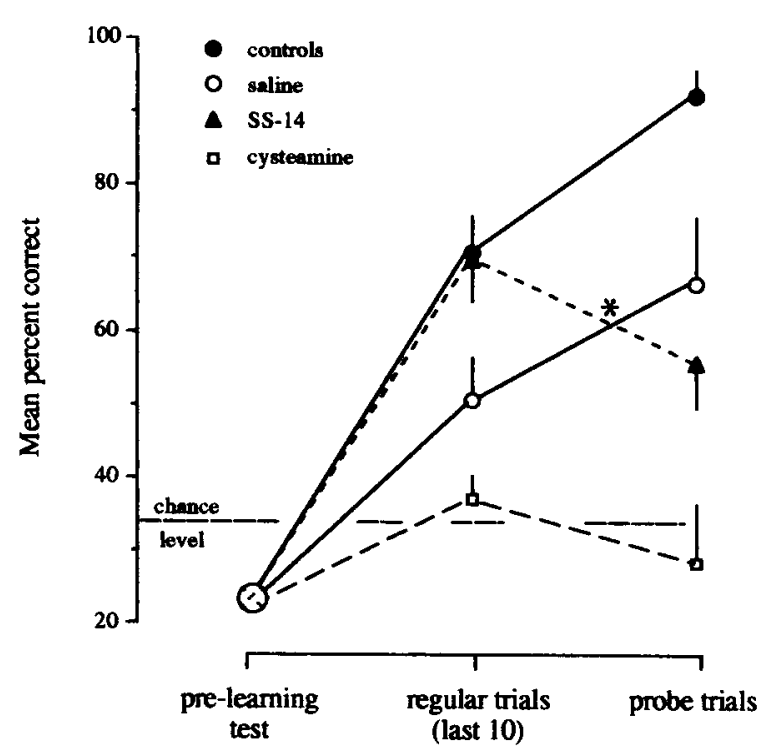

Figure 3. Performance (mean percent correct $\pm S E M$ ) on threechoice discrimination 10-trial blocks performed before learning (prelearning), on the last block of regular trials (mean transformed percent correct; see text); and on probe trials. *Group $\times$ trial interaction was significantly different from that of intact controls and saline $(p<.05)$. 
Table 1

Reference errors (mean $\pm S E M)$ following intrahippocampal infections of SS-14 (0.2 $\mu \mathrm{g})$ and of saline ( 9 animals per group), and in a routinely trained intact control group $(n=18)$, across seven sessions of the eight-arm discrimination task (Experiment 2).

\begin{tabular}{|c|c|c|c|c|c|c|c|}
\hline \multicolumn{8}{|c|}{ Sessions } \\
\hline Group & 1 & 2 & 3 & 4 & 5 & 6 & 7 \\
\hline Saline & $27.4 \pm 1.2$ & $29.3 \pm 1.4$ & $29.8 \pm 1.3$ & $22.0 \pm 1.6$ & $20.1 \pm 2.4$ & $14.8 \pm 2.7$ & $13.7 \pm 2.4$ \\
\hline SS-14 & $29.4 \pm 1.3$ & $30.0 \pm 1.2$ & $22.1 \pm 1.0^{\dagger}$ & $16.7 \pm 1.9^{*}$ & $14.1 \pm 2.0$ & $13.6 \pm 3.1$ & $9.0 \pm 1.7$ \\
\hline Controls & $29.6 \pm 1.3$ & $24.3 \pm 1.0$ & $20.4 \pm 1.8$ & $16.3 \pm 1.7$ & $13.1 \pm 1.4$ & $9.7 \pm 1.1$ & $9.2 \pm 1.1$ \\
\hline
\end{tabular}

*Significantly different from saline $(p<.05)$. †Significantly different from saline $(p<.01)$.

treatment group was impaired, thereby suggesting that either surgery (implanted cannulae), or intrahippocampal saline injections, or both factors, may be responsible for this deleterious behavioral effect (see Figure 2).

2. When testing was shifted from regular trials $(A-B+$ and $\mathrm{B}+\mathrm{C}-$ ) to probe trials (three-arm choice: $\mathrm{A}-\mathrm{B}+\mathrm{C}-$ ), somatostatin-injected mice no longer exhibited better choice accuracy than did saline-injected animals. In fact, whereas discrimination performance of both the saline and the intact control groups continued to progress from regular to probe trials, performance of the SS-14 group actually regressed (see Figure 3 ). All these points will be discussed in turn.

It seems likely that the impairment observed in the saline-treatment group resulted from the acute injection of the vehicle into the hippocampus, rather than from surgery and/or the lesion produced by the cannulae. Indeed, in a similar learning paradigm but with two distinct pairs of arms, no differences in acquisition rates were observed between a group of animals implanted with stimulating electrodes aimed at the same site as the cannulae and an intact control group (Demir \& Jaffard, unpublished observation). This would suggest that through an as-yetundefined mechanism, saline injection alone was sufficient to produce a hippocampal dysfunction that would be responsible for the slowing of the acquisition rate. Under these conditions, SS-14 would only-albeit almost completely-reverse the deficit produced by the saline injection, whereas its depletor, cysteamine, would almost completely prevent acquisition across the four sessions of regular trials. Since cysteamine has also been reported to decrease catecholamine levels in both the cortex and the striatum (Pitkänen et al., 1989), the large effects of the cysteamine injections may not only have been the simple consequence of SS-14 depletion. However, the opposite behavioral effects produced by SS-14 and cysteamine injections suggest a specific and unitary underlying pharmacological basis and also a specific involvement of hippocampal SS-14 neurons in this learning process. Subsequent testing on probe trials revealed, though, that the beneficial effect of SS-14 on regular trials cannot be considered to simply result from a facilitation of the same kind of memory processes as those underlying performance in both the saline and the intact control groups. More precisely, the observation that these two control groups behaved similarly when testing was shifted from regular to probe trials suggests that, even though weakened in the saline group, the memory processes that were engaged in each of these groups were qualitatively the same. In contrast, the present findings suggest that the somatostatin-injected mice had made their choices by using processes that were, at least in part, qualitatively different from those used by control mice. Thus, on the one hand, it seems likely that the use of elemental associations (i.e., Place B-food; Place A-no food; Place Cno food; see Rudy \& Sutherland, 1992) was sufficient to guide performance on both regular and probe trials. This strategy could thus account for performance in both control groups. On the other hand, the observation that somatostatin-treated mice were significantly less flexible than were controls when required to adapt to probe trials would suggest that they had used another strategy in the learning phase. One strategy that would allow correct responding on regular but not on probe trials might be the use of an egocentric conditional discrimination strategy in which each pair should be treated as a stimulus compound [i.e., if (AB), then go right; if (BC), then go left]. A similar hypothesis has been proposed to account for the specific impairment of fornix-lesioned rats on probe (vs. regular) discrimination trials in which previously learned odors (i.e., $\mathrm{A}+\mathrm{B}-, \mathrm{C}+\mathrm{D}-$ ) were presented in combinations not previously experienced (i.e., A+D-, B-C +; Eichenbaum, Mathews, \& Cohen, 1989). The authors assumed that poor performance of lesioned rats on probe trials could be accounted for by an impairment in the use of a "relational" strategy during initial learning (i.e., a strategy aimed at comparing events across trials and allowing subsequent adaptation to a previously nonencountered stimulus compound). In the present experiment, this would mean either that the B arm was not encoded as a place common to both pairs by the SS-14 group (i.e., these animals treated [ABC] as an unfamiliar compound), or, as recently suggested by Worden (1992), that these animals were unable to use efficient associative retrieval mechanisms aimed at fitting together the two "fragments" (AB) (BC) of information. Both hypotheses would adequately explain the impaired performance of somatostatin-injected mice on probe versus regular trials.

There is, however, a problem with these proposals. In Eichenbaum et al.'s (1989) experiment, lesioned rats were also impaired on original learning, whereas in the present one, SS-14 mice actually improved relative to the saline 
group. The facilitative effect of intrahippocampal somatostatin was confirmed in Experiment 2, in which a different spatial reference memory paradigm (three arms baited out of eight) was employed (see Table 1). Taken as a whole, the present results suggest that, irrespective of its abovementioned hypothesized effects on strategies, the peptide would facilitate place learning. Again, there are some problems with this proposal. If, for instance, one considers that, as stated by both Sutherland and Rudy (1989) and Eichenbaum (1992), spatial mapping processes (O'Keefe \& Nadel, 1978) are one important example of learning tasks relying on configural or relational processing, the presently observed facilitation produced by intrahippocampal somatostatin seems difficult to explain. Nevertheless, the observation that somatostatin facilitated LTP in mossy fiber-CA3 cell synapses through the intervention of cholinergic and noradrenergic neurons (Matsuoka et al., 1991a), whereas cysteamine reduced its magnitude (Matsuoka et al., 1991b), could account for the presently observed facilitative and disruptive effects of these compounds on spatial learning (Morris, 1989).

Developmental studies have provided evidence that, compared with adult rodents, infants (postnatal day 1221) exhibit four times the levels of hippocampal expression of preprosomatostatin mRNA (Bendotti et al., 1990; Fitzpatrick-McElligott, Card, O'Kane, \& Baldino, 1991). Thus, parallels between this rise in the SS-14 level and what is known about the memory function at this stage of development might be of interest with respect to the present results. It therefore seems important to note that infant rats (Postnatal Day 17-19) have been shown to display a marked propensity in encoding stimulus compounds (Mellon, Kraemer, \& Spear, 1991). Although indirect, this observation would be in agreement with the abovementioned postulated strategy used by the somatostatintreatment group during acquisition. However, the observation that place learning in the Morris water maze was actually impaired in infant rats up to 22 days of age (Rudy \& Paylor, 1988; Rudy, Stadier-Morris, \& Albert, 1987) again casts doubts upon the idea that a rise in SS-14 level is sufficient by itself to improve the ability to encode spatial information, as was suggested by the faster acquisition rates of SS-14 groups on both discrimination tasks. Finally, it is possible that the tasks that we used did not sufficiently tax spatial mapping or that facilitation of spatial learning may occur in the setting of a configural or relational learning impairment (see Gallagher \& Holland, 1992, for an inverse pattern of behavioral alterations following hippocampal damage). Further experiments are thus required, in order to identify the kinds of information processing that are sensitive to alterations in hippocampal somatostatin levels.

\section{REFERENCES}

BaKHIT, C., \& SWERdLow, N. (1986). Behavioral changes following central injection of cysteamine in rats. Brain Research, 365, 159-163.

Beal, M. F., Mazurek, M. F., Chattha, V. T., Bird, E. D., \& MARTIN, J. B. (1985). Reduced numbers of somatostatin receptors in the cerebral cortex in Alzheimer's disease. Science, 229, 289-291.
Bendott, C., Hohmann, C., Forloni, G., Reeves, R., Coyle, J. T., \& Oster-Granite, M. L. (1990). Developmental expression of somatostatin in mouse brain. II: In situ hybridization. Brain Research, 53, 26-39.

Bollók, I., Vécsei, L., \& TELEGdY, G. (1983). The effects between propranolol and somatostatin on active avoidance behavior, open-field activity and electro-convulsive-shock-induced amnesia of rats. Neuropeptides, 3, 263-270.

Brazeau, P., Vale, N., Burgus, R., Ling, N., Butcher, M., RivIER, J., \& GUILlEMIN, R. (1973). Hypothalamic polypeptide that inhibits the secretion of immunoreactive pituitary growth hormone. Science, 179, 77-79.

Chan-Palay, V. (1988). Somatostatin and neuropeptide Y alterations and coexistence in the Alzheimer's dementia hippocampus. Interdisciplinary Topics of Gerontology, 25, 38-58.

Davies, P., Katz, A., \& Crystal, H. A. (1982). Cholineacetyltransferase, somatostatin and substance $P$ in selected cases of Alzheimer's disease. In Alzheimer's disease: A report of progress. Aging, 19, 9-14.

Denoble, V. J., Hepler, D. J., \& Barto, R. A. (1989). Cysteamineinduced depletion of somatostatin produces differential cognitive deficits in rats. Brain Research, 482, 42-48.

Eichenbaum, H. (1992). The hippocampal system and declarative memory in animals. Journal of Cognitive Neuroscience, 4, 218-231.

Eichenbaum, H., Mathews, P., \& Cohen, N. (1989). Further studies of hippocampal representation during odor discrimination learning. Behavioral Neuroscience, 103, 1207-1216.

Eichenbaum H. T., Otto, T., Cohen, N. (1992). The hippocampus: What does it do? Behavioral \& Neural Biology, 57, 2-36.

Epelbaum, J., Brazeau, P., Tsang, D., BrowXer, J., \& Martin, J. B. (1977). Subcellular distribution of radioimmunoassayable somatostatin in rat brain. Brain Research, 126, 309-323.

Finley, J. C. W., Madedrut, J. L., Roger, L. J., \& Petrusz, P. (1981) The immunocytochemical localization of somatostatincontaining neurons in rat central nervous system. Neuroscience, 6 , 2173-2192.

FitzPatrick-McElligott, S., Card, J. P., O'Kane, T. M., \& BalDINo, F. J. R. (1991). Ontogeny of mRNA-containing perikarya in the central nervous system. Synapse, 7, 123-134.

Gallagher, M., \& Holland, P. C. (1992). Preserved configural learning and spatial learning impairment in rats with hippocampal damage. Hippocampus, 2, 81-88.

haroutunian, V., Mantin, R., Campbell, G.A., Tsuboyama, G. K., \& DAvis, K. L. (1987). Cysteamine-induced depletion of central somatostatin-like immunoactivity: Effect on behavior, learning, memory and brain neurochemistry. Brain Research, 403, 234-242.

IRITANI, S., \& SATOH, K. (1991). Distribution of somatostatinimmunoreactive cell bodies and fibers in the neocortex of macaca fuscata. Synapse, 9, 50-59.

Jaffard, R., Mocaer, E., Poignant, J. C., Michaeu, J., MarIGhetto, A., Meunier, M., \& Beracochea, D. (1991). Effects of tianeptine on spontaneous alternation, simple and concurrent spatial discrimination learning and on alcohol-induced alternation deficits in mice. Behavioural Pharmacology, 2, 37-46.

Katayama, S., Kito, S., Miyoshi, R., \& Yamamura, Y. (1990). Mapping of somatostatin receptor localization in rat brain: Forebrain and diencephalon. Brain Research, 24, 331-339.

KOHler, C., \& Chan-PAlay, V. (1982). Somatostatin-like immunoreactive neurons in the hippocampus: An immunocytochemical study in the rat. Neuroscience Letters, 34, 259-264.

KosaKa, T., WU, J. Y., \& BENoIT, R. (1988). GABA-ergic neurons containing somatostatin-like immunoreactivity in the rat hippocampus and dendate gyrus. Experimental Brain Research, 71, 388-398.

Matsuoka, N., KAneko, S., \& SATOH, M. (1991a). A facilitatory role of endogenous somatostatin in long-term potentiation of the mossy fiber-CA3 system in guinea-pig hippocampus. Neuroscience Letters, 129, 177-180.

Matsuoka, N., KaneKo, S., \& SAтoh, M. (1991b). Somatostatin augments long-term potentiation of the mossy fiber-CA3 system in guineapig hippocampal slice. Brain Research, 553, 188-194.

McCARTY, R., \& PlunketT, L. M. (1987). Quantitative autoradiographic analysis of somatostatin binding sites in discrete areas of rat forebrain. Brain Research Bulletin, 18, 29-34. 
Mellon, R. C., Kraemer, P. J., \& Spear, N. E. (1991). Intersensory development and Pavlovian conditioning stimulus selection and encoding of lights and tone in the preweanling rat. Journal of Experimental Psychology: Animal Behavior Processes, 17, 448-464.

MORRIS, R. G. M. (1989). Synaptic plasticity and learning: Selective impairment of learning in rats and blockade of long-term potentiation in vivo by N-Methyl-D-Aspartate receptor antagonist AP5. Journal of Neuroscience, 9, 3040-3057.

NADEL, L. (1991). The hippocampus and space revisited. Hippocampus, 1, 221-229.

OKEEFE, J., \& NADEL, L. (1978). The hippocampus as a cognitive map. Oxford: Oxford University Press Clarendon Press.

Olton, D. S., Becker, J. T., \& Handelman, G. E. (1979). Hippocampus, space and memory. Behavioral \& Brain Science, 2, 313-365.

Pitkänen, A., Valjakka, A., PÄ̈́kKonen, A., Sirvio, J., Jousmaki, V., Partanen, J., \& Riekkinen, P. J. (1989). Effect of cysteamine on levels of somatostatin-like immunoreactivity and catecholamines and on electroencephalogram in the rat brain. Neuropeptides, 14, 1-9.

Pittman, Q. J., Siggins, G. R. (1981). Somatostatin hyperpolarizes hippocampal pyramidal cells in vitro. Brain Research, 221, 402-408.

Reubi, J. C., \& MAURER, R. (1985). Autoradiographic mapping of somatostatin receptors in the rat central nervous system and pituitary. Neuroscience, 15, 1183-1193.

RezeK, M., Havlicek, V., Hughes, K. R., \& Friesen, H. (1976). Central site of somatostatin (SRIF): Role of the hippocampus. Neuropharmacology, 15, 499-504.

Rossor, M. N., Emson, P. C., Mountjoy, C. Q., Roth, M., \& IverSON, L. L. (1980). Reduced amounts of immunoreactive somatostatin in the temporal cortex in senile dementia of Alzheimer type. Neuroscience Letters, 20, 373-377.

RUdY, J. W., \& PAYLOR, R. (1988). Reducing the temporal demands of the Morris place-learning task fails to ameliorate the place-learning impairment of preweanling rats. Psychobiology, 16, 152-156.

Rudy, J. W., Stadler-Morris, S., \& Albert, P. (1987). Ontogeny of spatial navigation behaviors in the rat: Dissociation of "proximal" and "distal"'-cue based behaviors. Behavioral Neuroscience, 101, 62-73.

RudY, J. W., \&utherland, R. J. (1992). Configural and elemental associations and the memory coherence problem. Journal of Cognitive Neuroscience, 4, 208-216.

Sagar, S. M., Landry, C., Millard, W. J., Badger, T. M., ArNOLD, M. A., \& MARTIN, J. B. (1982). Depletion of somatostatinlike immunoreactivity in the rat central nervous system by cysteamine. Journal of Neuroscience, 2, 225-230.

Scharfman, H. E., \& Schwartzkroin, P. A. (1988). Further studies of the effects of somatostatin and related peptide in area CAl of rabbit hippocampus. Cellular \& Molecular Neurobiology, 8, 411-429.

Sutherland, R. J., \& Rudy, J. W. (1989). Configural association theory: The role of the hippocampal formation in learning, memory, and amnesia. Psychobiology, 17, 129-144.

VÉCSEI, L., BolLóK, I., \& TELEGDY, G. (1983). Intracerebroventricular somatostatin attenuates electroconvulsive shock-induced amnesia in rats. Peptides, 4, 293-295.

Vécsei, L., Kiraly, C., Bollók, I., Nagy, A., Varga, J., Penke, B., \& Telegdy, G. (1984). Comparative studies with somatostatin and cysteamine in different behavioral tests on rats. Pharmacology, Biochemistry \& Behavior, 21, 833-837.

VÉCSEI, L., \& WIDERLOW, E. (1988). Effects of intracerebroventricularly administered somatostatin on passive avoidance, shuttle-box behavior and open-field activity in rats. Neuropeptides, 12, 237-242.

WORDEN, R. (1992). Navigation by fragment fitting: A theory of hippocampal function. Hippocampus, 2, 165-168.

XIE, Z., \& SASTRY, B. R. (1992). Actions of somatostatin on GABAergic synaptic transmission in CA1 area of the hippocampus. Brain Research, 591, 239-247.

Yamano, M., Luiten, P. G. M. (1989). Direct synaptic contacts of medial septal efferents with somatostatin immunoreactive neurones in the rat hippocampus. Brain Research Bulletin, 22, 993-1001.

(Manuscript received March 31, 1993; revision accepted for publication June 30,1993 .) 